\title{
ON BIEBERBACH-EILENBERG FUNCTIONS. III
}

\author{
BY
}

JAMES A. JENKINS

1. In the earlier papers with this title [6], [7] we treated a number of extremal problems for the class of Bieberbach-Eilenberg functions, some of the results applying only when the functions were further required to be univalent. The method used was primarily that of considering appropriate module problems and applying circular symmetrization. In the present work we will first apply the generalized Area Principle to obtain various results for univalent BieberbachEilenberg functions. Secondly we will give an application of a somewhat different type of module problem which does not require the assumption of univalence. Similar applications are made in each case to a family of functions introduced by Shah [13]. The latter functions admit direct application of the General Coefficient Theorem, and we give an example of one such. It is then shown how a corresponding result can be obtained for Bieberbach-Eilenberg functions. Next we discuss ways in which the definition of both families can be generalized. Finally we remark on the manner in which the requirement of univalence can be removed for an earlier result.

2. We begin with the proof of the following basic result.

THEOREM 1. Let $f(z)$ be regular and univalent in $|z|<1$. Let $F(\zeta)$ be univalent in $|\zeta|>1$ and regular apart from a simple pole at the point at infinity where it has the Laurent expansion $F(\zeta)=c \zeta+c_{0}+c_{1} \zeta^{-1}+\cdots$. Let the images of $|z|<1$ by $w=f(z)$ and of $|\zeta|>1$ by $w=F(\zeta)$ be nonoverlapping. Let appropriate branches of the following functions have the expansions indicated, where $|z|,|Z|<1,|\zeta|,|\eta|>1$ :

$$
\begin{aligned}
\log \frac{f(z)-f(Z)}{z-Z} & =\sum_{m, l=0}^{\infty} A_{m l} z^{m} Z^{l}, \\
\log \left(1-\frac{f(z)}{F(\zeta)}\right) & =\sum_{m, l=1}^{\infty} \lambda_{m l^{m}} z^{m}, \\
\log \left(\frac{F(\zeta)-F(\eta)}{c(\zeta-\eta)}\right) & =\sum_{m, l=1}^{\infty} d_{m l} \zeta^{-m} \eta^{-l} .
\end{aligned}
$$

Let $z_{j}, j=1, \cdots, n, n>1$, be points in $|z|<1, \zeta_{k}, k=1, \cdots, N, N>0$, be points in $|\zeta|>1$. Let $\alpha_{j}, j=1, \cdots, n$, be complex constants satisfying $\sum_{j=1}^{n} \alpha_{j}=0$, $\mu_{k}, k=1, \cdots, N$, be arbitrary complex constants. Then

Received by the editors June 1, 1964. 


$$
\begin{aligned}
\sum_{m=1}^{\infty} m \mid \sum_{j=1}^{n} & \alpha_{j}\left(\sum_{l=1}^{\infty} A_{m l} z_{j}^{l}\right)+\left.\sum_{k=1}^{N} \mu_{k}\left(\sum_{l=1}^{\infty} \lambda_{m l} \zeta_{k}^{-l}\right)\right|^{2} \\
& +\sum_{l=1}^{\infty} l\left|\sum_{j=1}^{n} \alpha_{j}\left(\sum_{m=1}^{\infty} \lambda_{m l} z_{j}^{m}\right)+\sum_{k=1}^{N} \mu_{k}\left(\sum_{m=1}^{\infty} d_{m l} \zeta_{k}^{-m}\right)\right|^{2} \\
\leqq & -\sum_{j, j^{\prime}=1}^{n} \alpha_{j} \bar{\alpha}_{j^{\prime}} \log \left(1-z_{j} \bar{z}_{j^{\prime}}\right)-\sum_{k, k^{\prime}=1}^{N} \mu_{k} \bar{\mu}_{k^{\prime}} \log \left(1-\zeta_{k}^{-1} \zeta_{k^{\prime}}^{-1}\right)
\end{aligned}
$$

Let $r, \rho, 0<r<1,1<\rho<\infty$, be such that $z_{j}, j=1, \cdots, n$, lie in $|z|<r$ and $\zeta_{k}, k=1, \cdots, N$, lie in $|\zeta|>\rho$, let $E_{r}$ be the image of $|z|<r$ under $f$ with boundary $\Gamma_{r}$ and $G_{\rho}$ be the image of $|\zeta|>\rho$ under $F$ with boundary $\Lambda_{\rho}$, each boundary being given the counterclockwise sense. Let $w_{j}=f\left(z_{j}\right), j=1, \cdots, n, \omega_{k}=F\left(\zeta_{k}\right)$, $k=1, \cdots, N$. The complement $D_{r \rho}$ of $E_{r} \cup G_{\rho}$ has positive area in the metric

$$
\left|\sum_{j=1}^{n} \alpha_{j}\left(w-w_{j}\right)^{-1}+\sum_{k=1}^{N} \mu_{k}\left(w-\omega_{k}\right)^{-1}\right||d w| \text {. }
$$

Thus

$$
\iint_{D_{.} .}\left|\sum_{j=1}^{n} \alpha_{j}\left(w-w_{j}\right)^{-1}+\sum_{k=1}^{N} \mu_{k}\left(w-\omega_{k}\right)^{-1}\right|^{2} d A_{w}>0
$$

where $d A_{w}$ denotes the element of Euclidean area in the $w$-plane. We transform this into

$$
\begin{gathered}
\frac{1}{2 i}\left[-\int_{\Gamma_{r}}+\int_{\Lambda_{\rho}}\right]\left(\sum_{j=1}^{n} \bar{\alpha}_{j}\left(\log \left(w-w_{j}\right)\right)^{-}+\sum_{k=1}^{N} \bar{\mu}_{k}\left(\log \left(w-\omega_{k}\right)\right)^{-}\right) \\
\cdot d\left(\sum_{j=1}^{n} \alpha_{j} \log \left(w-w_{j}\right)+\sum_{k=1}^{N} \mu_{k} \log \left(w-\omega_{k}\right)\right)>0
\end{gathered}
$$

and moreover into

(2)

$$
\begin{aligned}
-\frac{1}{2 i} \int_{|z|=r}\left(\sum_{j=1}^{n} \bar{\alpha}_{j}\left(\log \left(f(z)-f\left(z_{j}\right)\right)\right)^{-}+\sum_{k=1}^{N} \bar{\mu}_{k}\left(\log \left(f(z)-F\left(\zeta_{k}\right)\right)\right)^{-}\right) \\
\cdot d\left(\sum_{j=1}^{n} \alpha_{j} \log \left(f(z)-f\left(z_{j}\right)\right)+\sum_{k=1}^{N} \mu_{k} \log \left(f(z)-F\left(\zeta_{k}\right)\right)\right) \\
+\frac{1}{2 i} \int_{|\zeta|=\rho}\left(\sum_{j=1}^{n} \bar{\alpha}_{j}\left(\log \left(F(\zeta)-f\left(z_{j}\right)\right)\right)^{-}+\sum_{k=1}^{N} \bar{\mu}_{k}\left(\log \left(F(\zeta)-F\left(\zeta_{k}\right)\right)\right)^{-}\right) \\
\cdot d\left(\sum_{j=1}^{n} \alpha_{j} \log \left(F(\zeta)-f\left(z_{j}\right)\right)+\sum_{k=1}^{N} \mu_{k} \log \left(F(\zeta)-F\left(\zeta_{k}\right)\right)\right)>0 .
\end{aligned}
$$

Now we have on $|z|=r$ the Laurent expansion 


$$
\begin{aligned}
\sum_{j=1}^{n} & \alpha_{j} \log \left(f(z)-f\left(z_{i}\right)\right)+\sum_{k=1}^{N} \mu_{k} \log \left(f(z)-F\left(\zeta_{k}\right)\right) \\
= & \sum_{j=1}^{n} \alpha_{j} \log \left(\frac{f(z)-f\left(z_{j}\right)}{z-z_{j}}\right)-\sum_{j=1}^{n} \alpha_{j} \log \left(1-\frac{z_{j}}{z}\right)+\sum_{k=1}^{N} \mu_{k} \log \left(1-\frac{f(z)}{F\left(\zeta_{k}\right)}\right) \\
& + \text { constant } \\
= & \sum_{j=1}^{n} \alpha_{j}\left(\sum_{m, l=0}^{\infty} A_{m l} z^{m} z_{j}^{l}-\sum_{q=1}^{\infty} \frac{1}{q}\left(\frac{z_{j}}{z}\right)^{q}\right)+\text { constant }+\sum_{k=1}^{N} \mu_{k} \sum_{m, l=1}^{\infty} \lambda_{m l} z^{m} \zeta_{k}^{-l} .
\end{aligned}
$$

Also we have on $|\zeta|=\rho$ the Laurent expansion

$$
\begin{aligned}
\sum_{j=1}^{n} & \alpha_{j} \log \left(F(\zeta)-f\left(z_{j}\right)\right)+\sum_{k=1}^{N} \mu_{k} \log \left(F(\zeta)-F\left(\zeta_{k}\right)\right) \\
= & \sum_{j=1}^{n} \alpha_{j} \log \left(1-\frac{f\left(z_{j}\right)}{F(\zeta)}\right)+\sum_{k=1}^{N} \mu_{k} \log \left(\frac{F(\zeta)-F\left(\zeta_{k}\right)}{c\left(\zeta-\zeta_{k}\right)}\right)+\sum_{k=1}^{N} \mu_{k} \log \left(1-\frac{\zeta}{\zeta_{k}}\right) \\
& + \text { constant } \\
= & \sum_{j=1}^{n} \alpha_{j} \sum_{m, l=1}^{\infty} \lambda_{m l} z_{j}^{m} \zeta^{-l}+\sum_{k=1}^{N} \mu_{k}\left(\sum_{m, l=1}^{\infty} d_{m l} \zeta_{k}^{-m} \zeta^{-l}-\sum_{q=1}^{\infty} \frac{1}{q}\left(\frac{\zeta}{\zeta_{k}}\right)^{q}\right) \\
& + \text { constant. }
\end{aligned}
$$

Inserting these expansions in inequality (2), performing the indicated integrations and dividing by $\pi$ we obtain

$$
\begin{aligned}
\sum_{m=1}^{\infty} m & \left|\sum_{j=1}^{n} \alpha_{j}\left(\sum_{l=1}^{\infty} A_{m l} z_{j}^{l}\right)+\sum_{k=1}^{N} \mu_{k}\left(\sum_{l=1}^{\infty} \lambda_{m l} \zeta_{k}^{-l}\right)\right|{ }^{2} r^{2 m} \\
& +\sum_{l=1}^{\infty} l\left|\sum_{j=1}^{n} \alpha_{j}\left(\sum_{m=1}^{\infty} \lambda_{m l} z_{j}^{m}\right)+\sum_{k=1}^{N} \mu_{k}\left(\sum_{m=1}^{\infty} d_{m l} \zeta_{k}^{-m}\right)\right|^{2} \rho^{-2 l} \\
& \leqq \sum_{q=1}^{\infty} \frac{1}{q}\left|\sum_{j=1}^{n} \alpha_{j} z_{j}^{q}\right|^{2} r^{-2 q}+\sum_{q=1}^{\infty} \frac{1}{q}\left|\sum_{k=1}^{N} \mu_{k} \zeta_{k}^{-q}\right|^{2} \rho^{2 q}
\end{aligned}
$$

Letting $r$ and $\rho$ tend to 1 and performing an explicit reduction of the right-hand side we have

$$
\begin{aligned}
\sum_{m=1}^{\infty} m \mid & \sum_{j=1}^{n} \alpha_{j}\left(\sum_{l=1}^{\infty} A_{m l} z_{j}^{l}\right)+\left.\sum_{k=1}^{N} \mu_{k}\left(\sum_{l=1}^{\infty} \lambda_{m l} \zeta_{k}^{-l}\right)\right|^{2} \\
& +\sum_{l=1}^{\infty} l\left|\sum_{i=1}^{n} \alpha_{j}\left(\sum_{m=1}^{\infty} \lambda_{m l} z_{j}^{m}\right)+\sum_{k=1}^{N} \mu_{k}\left(\sum_{m=1}^{\infty} d_{m l} \zeta_{k}^{-m}\right)\right|^{2} \\
\leqq & -\sum_{j, j^{\prime}=1}^{n} \alpha_{j} \bar{\alpha}_{j^{\prime}} \log \left(1-z_{j} \bar{z}_{j^{\prime}}\right)-\sum_{k, k^{\prime}=1}^{N} \mu_{k} \bar{\mu}_{k^{\prime}} \log \left(1-\zeta_{k}^{-1} \bar{\zeta}_{k^{\prime}}^{-1}\right)
\end{aligned}
$$

This is just the inequality (1). 
COROllary 1. Using the terminology of Theorem 1, let in addition $Z_{p}$, $p=1, \cdots, t, t>1$, be points in $|z|<1, \eta_{q}, q=1, \cdots, T, T>0$, be points in $|\zeta|>1$. Let $\beta_{p}, p=1, \cdots, t$, be complex constants satisfying $\sum_{p=1}^{t} \beta_{p}=0$, $v_{q}, q=1, \cdots, T$, be arbitrary complex constants. Then

$$
\begin{aligned}
& \left(-\sum_{p, p^{\prime}=1}^{t} \beta_{p} \bar{\beta}_{p^{\prime}} \log \left(1-Z_{p^{\prime}} Z_{p^{\prime}}\right)\right)^{-1} \\
& \cdot\left|\sum_{j=1}^{n} \sum_{p=1}^{t} \alpha_{j} \beta_{p} \log \frac{f\left(z_{j}\right)-f\left(Z_{p}\right)}{z_{j}-Z_{p}}+\sum_{p=1}^{t} \sum_{k=1}^{N} \beta_{p} \mu_{k} \log \left(1-\frac{f\left(Z_{p}\right)}{F\left(\zeta_{k}\right)}\right)\right|^{2} \\
& \quad+\left(-\sum_{q, q^{\prime}=1}^{T} v_{q} \bar{v}_{q^{\prime}} \log \left(1-\eta_{q}^{-1} \bar{\eta}_{q^{\prime}}^{-1}\right)\right)^{-1} \\
& \quad \cdot\left|\sum_{j=1}^{n} \sum_{q=1}^{T} \alpha_{j} v_{q} \log \left(1-\frac{f\left(z_{j}\right)}{F\left(\eta_{q}\right)}\right)+\sum_{k=1}^{N} \sum_{q=1}^{T} \mu_{k} v_{q} \log \left(\frac{F\left(\zeta_{k}\right)-F\left(\eta_{q}\right)}{c\left(\zeta_{k}-\eta_{q}\right)}\right)\right|^{2} \\
& \leqq \\
& -\sum_{j, j^{\prime}=1}^{n} \alpha_{j} \bar{\alpha}_{j^{\prime}} \log \left(1-z_{j^{\prime}} \bar{z}_{j^{\prime}}\right)-\sum_{k, k^{\prime}=1}^{N} \mu_{k} \bar{\mu}_{k^{\prime}} \log \left(1-\zeta_{k}^{-1} \bar{\zeta}_{k^{\prime}}^{-1}\right) .
\end{aligned}
$$

Consider

$$
\left|\sum_{j=1}^{n} \sum_{p=1}^{t} \alpha_{j} \beta_{p} \log \frac{f\left(z_{j}\right)-f\left(Z_{p}\right)}{z_{j}-Z_{p}}+\sum_{p=1}^{t} \sum_{k=1}^{N} \beta_{p} \mu_{k} \log \left(1-\frac{f\left(Z_{p}\right)}{F\left(\zeta_{k}\right)}\right)\right|^{2}
$$

which can be written as the following expression and thus evidently satisfies the inequality

$$
\begin{aligned}
& \left|\sum_{j=1}^{n} \sum_{p=1}^{l} \alpha_{j} \beta_{p} \sum_{m, l=1}^{\infty} A_{m l} Z_{p}^{m} z_{j}^{l}+\sum_{p=1}^{t} \sum_{k=1}^{N} \beta_{p} \mu_{k} \sum_{m, l=1}^{\infty} \lambda_{m l} Z_{p}^{m} \zeta_{k}^{-l}\right|^{2} \\
& \leqq-\left(\sum_{m=1}^{\infty} \frac{1}{m}\left|\sum_{p=1}^{t} \beta_{p} Z_{p}^{m}\right|^{2}\right) \\
& \quad \cdot\left(\sum_{m=1}^{\infty} m\left|\sum_{j=1}^{n} \alpha_{j}\left(\sum_{l=1}^{\infty} A_{m l} z_{j}^{l}\right)+\sum_{k=1}^{N} \mu_{k}\left(\sum_{l=1}^{\infty} \lambda_{m l} \zeta_{k}^{-l}\right)\right|^{2}\right) .
\end{aligned}
$$

Similarly

$$
\begin{aligned}
&\left|\sum_{j=1}^{n} \sum_{q=1}^{T} \alpha_{j} v_{q} \log \left(1-\frac{f\left(z_{j}\right)}{F\left(\eta_{q}\right)}\right)+\sum_{k=1}^{N} \sum_{q=1}^{T} \mu_{k} v_{q} \log \left(\frac{F\left(\zeta_{k}\right)-F\left(\eta_{q}\right)}{c\left(\zeta_{k}-\eta_{q}\right)}\right)\right|^{2} \\
&=\left|\sum_{j=1}^{n} \sum_{q=1}^{T} \alpha_{j} v_{q} \sum_{m, l=1}^{\infty} \lambda_{m l} z_{j}^{m} \eta_{q}^{-l}+\sum_{k=1}^{N} \sum_{q=1}^{T} \mu_{k} v_{q} \sum_{m, l=1}^{\infty} d_{m l} \zeta_{k}^{-m} \eta_{q}^{-l}\right|^{2} \\
& \leqq\left(\sum_{l=1}^{\infty} \frac{1}{l}\left|\sum_{q=1}^{T} v_{q} \eta_{q}^{-l \mid}\right|^{2}\right) \\
&\left(\sum_{l=1}^{\infty}\left|\sum_{j=1}^{n} \alpha_{j}\left(\sum_{m=1}^{\infty} \lambda_{m l} z_{j}^{m}\right)+\sum_{k=1}^{N} \mu_{k}\left(\sum_{m=1}^{\infty} d_{m l} \zeta_{k}^{-m}\right)\right|^{2}\right)
\end{aligned}
$$


Combining these inequalities, using the evident reduction of the first term on the right-hand side in each case and employing inequality (1) we obtain at once inequality (4).

Inequality (4) can be reduced to various other forms, but we will confine ourselves to considering the following special case.

COROLlary 2. Let $f(z)$ be regular and univalent for $|z|<1$ with $f(0)=0$. Let $F(\zeta)$ be univalent in $|\zeta|>1$ and regular apart from a simple pole at the point at infinity where it has the Laurent expansion $F(\zeta)=c \zeta+c_{0}+c_{1} \zeta^{-1}+\cdots$. Let the images of $|z|<1$ by $w=f(z)$ and of $|\zeta|>1$ by $w=F(\zeta)$ be nonoverlapping. Then for $|z|,|Z|<1,|\zeta|,|\eta|>1, \alpha, \mu$ arbitrary complex numbers we have

$$
\begin{aligned}
& {\left[-\log \left(1-|Z|^{2}\right)\right]^{-1}\left|\alpha \log \frac{f^{\prime}(0) z Z(f(z)-f(Z))}{f(z) f(Z)(z-Z)}+\mu \log \left(1-\frac{f(Z)}{F(\zeta)}\right)\right|^{2}} \\
& \quad+\left[-\log \left(1-|\eta|^{-2}\right)\right]^{-1}\left|\alpha \log \left(1-\frac{f(z)}{F(\eta)}\right)+\mu \log \frac{(F(\zeta)-F(\eta))}{c(\zeta-\eta)}\right|^{2} \\
& \leqq-|\alpha|^{2} \log \left(1-|z|^{2}\right)-|\mu|^{2} \log \left(1-|\zeta|^{-2}\right) .
\end{aligned}
$$

This is obtained by setting in inequality (4), $n=t=2, N=T=1, z_{2}=Z_{2}=0$ and writing $z, Z, \zeta, \eta, \alpha, \mu$ for $z_{1}, Z_{1}, \zeta_{1}, \eta_{1}, \alpha_{1}=-\alpha_{2}, \mu_{1}$.

If in inequality (5) we take $z=Z, \zeta=\eta$ (using limiting values where appropriate) and apply Schwarz's inequality, we obtain

$$
\begin{aligned}
\mid \alpha^{2} \log \frac{z^{2} f^{\prime}(0) f^{\prime}(z)}{(f(z))^{2}} & +2 \alpha \mu \log \left(1-\frac{f(z)}{F(\zeta)}\right)+\mu^{2} \log c^{-1} F^{\prime}(\zeta) \mid \\
& \leqq-|\alpha|^{2} \log \left(1-|z|^{2}\right)-|\mu|^{2} \log \left(1-|\zeta|^{-2}\right)
\end{aligned}
$$

an inequality due to Alenitsyn [1].

Corollary 3. Under the assumptions of Corollary 2

$$
\log \left(1-\frac{f(z)}{F(\eta)}\right) \leqq\left[\log \left(1-|z|^{2}\right) \log \left(1-|\eta|^{-2}\right)\right]^{1 / 2}
$$

This is obtained by taking $\alpha=1, \mu=0$ in inequality (5). It improves Alenitsyn's bound $-\frac{1}{2}\left[\log \left(1-|z|^{2}\right)+\log \left(1-|\eta|^{-2}\right)\right]$.

3. We denote by $C$ the class of functions $f(z)$ regular for $|z|<1$ which satisfy $f(0)=0$ and the condition $f\left(z_{1}\right) f\left(z_{2}\right) \neq 1,\left|z_{1}\right|<1,\left|z_{2}\right|<1$. We denote by $C^{*}$ the subclass of $C$ consisting of univalent functions.

Corollary 4. Let $f(z) \in C^{*}$. Let $\left|z_{j}\right|<1, j=1, \cdots, n,\left|x_{k}\right|<1, k=1, \cdots, N$, $\left|Z_{p}\right|<1, \quad p=1, \cdots, t, \quad\left|X_{q}\right|<1, q=1, \cdots, T$. Let $\alpha_{j}, j=1, \cdots, n$, be complex constants satisfying $\sum_{j=1} \alpha_{j}=0, \beta_{p}, p=1, \cdots, t$, be complex constants satisfying $\sum_{p=1}^{t} \beta_{p}=0, \mu_{k}, k=1, \cdots, N, v_{q}, q=1, \cdots, T$, be arbitrary complex constants. 
Then

(7)

$$
\begin{aligned}
& \left(-\sum_{p, p^{\prime}=1}^{t} \beta_{p} \bar{\beta}_{p^{\prime}} \log \left(1-Z_{p} Z_{p^{\prime}}\right)\right)^{-1} \\
& \cdot \mid \sum_{j=1}^{n} \sum_{p=1}^{t} \alpha_{j} \beta_{p} \log \frac{f\left(z_{j}\right)-f\left(Z_{p}\right)}{z_{j}-Z_{p}}+\sum_{p=1}^{t} \sum_{k=1}^{N} \beta_{p} \mu_{k} \log \left(1-f\left(\left.Z_{p}\left(f\left(x_{k}\right)\right)\right|^{2}\right.\right. \\
& +\left(-\sum_{q, q^{\prime}=1}^{T} v_{q} \bar{v}_{q^{\prime}} \log \left(1-X_{q} \bar{X}_{q^{\prime}}\right)\right)^{-1}
\end{aligned}
$$

$$
\begin{gathered}
\cdot\left|\sum_{j=1}^{n} \sum_{q=1}^{T} \alpha_{j} v_{q} \log \left(1-f\left(z_{j}\right) f\left(X_{q}\right)\right)+\sum_{k=1}^{N} \sum_{q=1}^{T} \mu_{k} v_{q} \log \left(\frac{f^{\prime}(0) x_{k} X_{q}\left(f\left(x_{k}\right)-f\left(X_{q}\right)\right)}{f\left(x_{k}\right) f\left(X_{q}\right)\left(x_{k}-X_{q}\right)}\right)\right|^{2} \\
\leqq-\sum_{j, j^{\prime}=1}^{n} \alpha_{j} \bar{\alpha}_{j^{\prime}} \log \left(1-z_{j} \bar{z}_{j^{\prime}}\right)-\sum_{k, k^{\prime}=1}^{N} \mu_{k} \bar{\mu}_{k^{\prime}} \log \left(1-x_{k} \bar{x}_{k^{\prime}}\right) .
\end{gathered}
$$

This is obtained by setting $F(\zeta)=\left(f\left(\zeta^{-1}\right)\right)^{-1}, \zeta^{-1}=x, \eta^{-1}=X$ in Corollary 1 . Corollary 5. Let $f(z) \in C^{*}$, let $|z|,|Z|,|x|,|X|<1$, let $\alpha, \mu$ be arbitrary complex constants. Then

$$
\left[-\log \left(1-|Z|^{2}\right)\right]^{-1}\left|\alpha \log \frac{f^{\prime}(0) z Z(f(z)-f(Z))}{f(z) f(Z)(z-Z)}+\mu \log (1-f(Z) f(x))\right|^{2}
$$

$$
\begin{aligned}
& +\left[-\log \left(1-|X|^{2}\right)\right]^{-1}\left|\alpha \log (1-f(z) f(X))+\mu \log \frac{f^{\prime}(0) x X(f(x)-f(X))}{f(x) f(X)(x-X)}\right|^{2} \\
\leqq & -|\alpha|^{2} \log \left(1-|z|^{2}\right)-|\mu|^{2} \log \left(1-|x|^{2}\right) .
\end{aligned}
$$

This is obtained by setting $F(\zeta)=\left(f\left(\zeta^{-1}\right)\right)^{-1}, \zeta^{-1}=x, \eta^{-1}=X$ in Corollary 2. Corollary 6. Let $f(z) \in C^{*}$, let $|z|,|Z|<1$. Then

$$
\begin{gathered}
\left.|\log (1-f(z) f(Z))| \leqq\left[\log \left(1-|z|^{2}\right) \log \left(1-|Z|^{2}\right)\right]^{1 / 2}\right) \\
\left|\log \frac{f^{\prime}(0) z Z(f(z)-f(Z))}{f(z) f(Z)(z-Z)} \pm \log (1-f(z) f(Z))\right| \\
\leqq\left[\log \left(1-|z|^{2}\right) \log \left(1-|Z|^{2}\right)\right]^{1 / 2}
\end{gathered}
$$

These results are obtained by setting $X=Z, x=z$ in (8) and for (9) setting $\alpha=0, \mu=1$, for (10) setting respectively $\alpha=\mu=1$ and $\alpha=-\mu=1$. They generalize results of Alenitsyn.

We denote by $K$ the class of functions $f(z)$ regular for $|z|<1$ and such that $f(0)=0, f\left(z_{1}\right) \bar{f}\left(z_{2}\right) \neq-1,\left|z_{1}\right|,\left|z_{2}\right|<1$. We denote by $K^{*}$ the subclass of $K$ consisting of univalent functions.

Corollary 7. Let $f(z) \in K^{*}$. Let $\left|z_{j}\right|<1, j=1, \cdots, n, \quad\left|x_{k}\right|<1, k=1, \cdots, N$, $\left|Z_{p}\right|<1, p=1, \cdots, t, \quad\left|X_{q}\right|<1, q=1, \cdots, T$. Let $\alpha_{j}, j=1, \cdots, n$, be complex 
constants satisfying $\sum_{j=1}^{n} \alpha_{j}=0, \beta_{p}, p=1, \cdots, t$, be complex constants satisfying $\sum_{p=1}^{t} \beta_{p}=0, \mu_{k}, k=1, \cdots, N, v_{q}, q=1, \cdots, T$, be arbitrary complex constants. Then

$$
\begin{aligned}
& \left(-\sum_{p, p^{\prime}=1}^{t} \beta_{p} \bar{\beta}_{p^{\prime}} \log \left(1-Z_{p} Z_{p^{\prime}}\right)\right)^{-1} \\
& \quad \cdot\left|\sum_{j=1}^{n} \sum_{p=1}^{t} \alpha_{j} \beta_{p} \log \frac{f\left(z_{j}\right)-f\left(Z_{p}\right)}{z_{j}-Z_{p}}+\sum_{p=1}^{t} \sum_{k=1}^{N} \beta_{p} \mu_{k} \log \left(1+f\left(Z_{p}\right) \bar{f}\left(x_{k}\right)\right)\right|^{2} \\
& \quad+\left(-\sum_{q, q^{\prime}=1}^{T} v_{q} \bar{v}_{q^{\prime}} \log \left(1-X_{q} \bar{X}_{q^{\prime}}\right)\right)^{-1} \mid \sum_{j=1}^{n} \sum_{q=1}^{T} \alpha_{j} v_{q} \log \left(1+f\left(z_{j}\right) \tilde{f}\left(X_{q}\right)\right) \\
& \quad+\left.\sum_{k=1}^{N} \sum_{q=1}^{T} \mu_{k} v_{q} \log \left(\frac{\left(\overline { f } ^ { \prime } ( 0 ) \overline { x } _ { k } \overline { X } _ { q } \left(\bar{f}\left(x_{k}\right)-\bar{f}\left(X_{q}\right)\right.\right.}{\tilde{f}^{\prime}\left(x_{k}\right) \tilde{f}^{\prime}\left(X_{q}\right)\left(\bar{x}_{k}-\bar{X}_{q}\right)}\right)\right|^{2} \\
& \leqq-\sum_{j, j^{\prime}=1}^{n} \alpha_{j} \bar{\alpha}_{j^{\prime}} \log \left(1-z_{j^{\prime}} \bar{z}_{j^{\prime}}\right)-\sum_{k, k^{\prime}=1}^{N} \mu_{k} \bar{\mu}_{k^{\prime}} \log \left(1-x_{k} \bar{x}_{k^{\prime}}\right) .
\end{aligned}
$$

This is obtained by setting $F(\zeta)=-\left(\bar{f}\left(\bar{\zeta}^{-1}\right)\right)^{-1}, \bar{\zeta}^{-1}=x, \bar{\eta}^{-1}=X$ in Corollary 1.

CoRollary 8. Let $f(z) \in K^{*}$, let $|z|,|Z|,|x|,|X|<1$, let $\alpha, \mu$ be arbitrary complex constants. Then

$$
\begin{aligned}
& {\left[-\log \left(1-|Z|^{2}\right)\right]^{-1}\left|\alpha \log \frac{f^{\prime}(0) z Z(f(z)-f(Z))}{f(z) f(Z)(z-Z)}+\mu \log (1+\bar{f}(Z) f(x))\right|^{2}} \\
& +\left[-\log \left(1-|X|^{2}\right)\right]^{-1}\left|\alpha \log (1+f(z) \tilde{f}(X))+\mu \log \frac{\bar{f}^{\prime}(0) \bar{x} \bar{X}(\bar{f}(x)-\bar{f}(X))}{\tilde{f}^{2}(x) \dot{f} \bar{f}(X)(\bar{x}-\bar{X})}\right| \\
& \leqq-|\alpha|^{2} \log \left(1-|z|^{2}\right)-|\mu|^{2} \log \left(1-|x|^{2}\right) .
\end{aligned}
$$

This is obtained by setting $F(\zeta)=-\left(\bar{f}\left(\zeta^{-1}\right)\right)^{-1}, \zeta^{-1}=x, \bar{\eta}^{-1}=X$ in Corollary 2.

Corollary 9. Let $f(z) \in K^{*}$, let $|z|,|Z|<1$. Then

$$
\begin{gathered}
|\log (1+f(z) \bar{f}(Z))| \leqq\left[\log \left(1-|z|^{2}\right) \log \left(1-|Z|^{2}\right)\right]^{1 / 2}, \\
\left|\log \frac{f^{\prime}(0) z Z(f(z)-f(Z))}{f(z) f(Z)(z-Z)} \pm \log (1+\bar{f}(z) f(Z))\right| \\
\leqq\left[\log \left(1-|z|^{2}\right) \log \left(1-|Z|^{2}\right)\right]^{1 / 2}
\end{gathered}
$$

These results are obtained by setting $X=Z, x=z$ in (12) and for (13) setting $\alpha=1, \mu=0$, for (14) setting respectively $\alpha=\mu=1, \alpha=-\mu=1$.

COROLlary 10. Let $f(z) \in K$. Then, for $|z|<1$,

$$
|f(z)| \leqq|z|\left(1-|z|^{2}\right)^{-1 / 2} \text {. }
$$

This result is obtained for $f(z) \in K^{*}$ by setting $z=Z$ in (13) to get

$$
\log \left(1+|f(z)|^{2}\right) \leqq-\log \left(1-|z|^{2}\right)
$$


and reducing by elementary means. For $f(z) \in K$ the result follows by the principle of subordination. Earlier proofs of this result use quite different techniques [9], [13].

It should be remarked that Lebedev [12] has also applied the Area Principle to the families $C, C^{*}, K, K^{*}$. His method is slightly different technically. Reduced to the present context it would give an inequality corresponding to (4) but with the assumption $n=t=N+1=T+1$. His problems for the above classes are not those treated here.

4. It is possible also to treat questions of the same general form as those in Theorem 1 by the traditional techniques of the method of the extremal metric. One is naturally led to a different form of inequality. Of particular interest is the fact that it is not necessary to require univalence of the functions involved.

THEOREM 2. Let $f(z)$ be meromorphic in $|z|<1$. Let $F(\zeta)$ be meromorphic in $|\zeta|>1$. Let the images of $|z|<1$ by $w=f(z)$ and of $|\zeta|>1$ by $w=F(\zeta)$ be nonoverlapping (as point sets). Let $z_{1}, \cdots, z_{n}$ be (distinct) points in $|z|<1$, and $\zeta_{1}, \cdots, \zeta_{N}$ be (distinct) finite points in $|\zeta|>1$. Let $a_{j}, j=1, \cdots, n, \beta_{k}$, $k=1, \cdots, N$, be positive constants such that $\sum_{j=1}^{n} \alpha_{j}=\sum_{k=1}^{N} \beta_{k}$. Denote $P(u, v)=(u-v)(1-\bar{v} u)^{-1}$. Then

$$
\begin{aligned}
& \prod_{j=1}^{n}\left|f^{\prime}\left(z_{j}\right)\right|^{\alpha_{j}^{2}} \prod_{k=1}^{N}\left|F^{\prime}\left(\zeta_{k}\right)\right|^{\mid \beta_{k}^{2}} \prod_{j \neq l ; j, l}\left|f\left(z_{j}\right)-f\left(z_{l}\right)\right|^{2 \alpha_{j} \alpha_{l}} \\
& \cdot \prod_{k \neq m: k, m}\left|F\left(\zeta_{k}\right)-F\left(\zeta_{m}\right)\right|^{2 \beta_{k} \beta_{m}} \prod_{j=1}^{n} \prod_{k=1}^{N} f\left(z_{j}\right)-\left.F\left(\zeta_{k}\right)\right|^{-2 \alpha_{j} \beta_{k}} \\
& \leqq \prod_{j \neq l: j, l}\left|P\left(z_{j}, z_{l}\right)\right|^{2 \alpha_{j} \alpha_{l}} \prod_{k \neq m ; k, m}\left|P\left(\zeta_{k}, \zeta_{m}\right)\right|^{2 \beta_{k} \beta_{m}} \prod_{j=1}^{n}\left(1-\left|z_{j}\right|^{2}\right)^{-\alpha_{j}^{2}} \\
& \cdot \prod_{k=1}^{N}\left(1-\left|\zeta_{k}\right|^{2}\right)^{-\beta_{k}^{2}} .
\end{aligned}
$$

This formula applies to the case where none of $f\left(z_{j}\right), j=1, \cdots, n, \zeta_{k}, F\left(\zeta_{k}\right)$, $k=1, \cdots, N$, is the point at infinity. In case one or more of these exclusions fails, an appropriately modified formula replaces (15).

In $|z| \leqq 1$ we regard the function

$$
U(z)=\log \left(\prod_{j=1}^{n}\left|P\left(z, z_{j}\right)\right|^{\alpha_{j}}\right)
$$

which is equal to zero on $|z|=1$, negative in $|z|<1$ and with negative logarithmic poles at the points $z_{j}, j=1, \cdots, n$. The level set $U_{t}$ consisting of points where $U(z)=-t(t>0)$, if we exclude a finite number of values of $t$ corresponding to critical points, consists of a finite number of Jordan curves which, together with $|z|=1$, bound a multiply-connected domain $D_{t}$ containing none of $z_{j}$, 
$j=1, \cdots, n$. Let $\Gamma$ denote the family of sets of Jordan curves which have this property and $\Gamma_{t}$ its subfamily for which the curves lie in $D_{t}$. Let the module of $D_{t}$ for the family of curve sets $\Gamma_{t}$ be denoted by $M_{t}$. It is well known that, taken in $L$ normalization $\left[9\right.$, p. 14], the function $\left(2 \pi \sum_{j=1}^{n} \alpha_{j}\right)^{-1}|\operatorname{grad} U(z)|$ is extremal for this module problem and that

$$
M_{t}=\left(2 \pi \sum_{j=1}^{n} \alpha_{j}\right)^{-1} t
$$

In $|\zeta| \geqq 1$ we regard the function

$$
V(\zeta)=\log \left(\prod_{k=1}^{N}\left|P\left(\zeta, \zeta_{k}\right)\right|^{-\beta_{k}}\right)
$$

which is equal to zero on $|\zeta|=1$, positive in $|\zeta|>1$ and with positive logarithmic poles at the points $\zeta_{k}, k=1, \cdots, N$. The level set $V_{s}$ consisting of points where $V(\zeta)=s(s>0)$, if we exclude a finite number of values of $s$ corresponding to critical points, consists of a finite number of Jordan curves which, together with $|\zeta|=1$, bound (on the $\zeta$-sphere) a multiply-connected domain $\Delta_{s}$ containing none of $\zeta_{k}, k=1, \cdots, N$. Let $\Lambda$ denote the family of sets of Jordan curves which have this property and $\Lambda_{s}$ its subfamily for which the curves lie in $\Delta_{s}$. Let the module of $\Delta_{s}$ for the family of curve sets $\Lambda_{s}$ be denoted by $N_{s}$. As above, taken in $L$-normalization, the function $\left(2 \pi \sum_{k=1}^{N} \beta_{k}\right)^{-1}|\operatorname{grad} V(\zeta)|$ is extremal for this module problem and

$$
N_{s}=\left(2 \pi \sum_{k=1}^{N} \beta_{k}\right)^{-1} s
$$

For convenience let us write $w_{j}=f\left(z_{j}\right), j=1, \cdots, n, \omega_{k}=F\left(\zeta_{k}\right), k=1, \cdots, N$. We now consider the function

$$
W(w)=\log \left(\prod_{j=1}^{n}\left|w-w_{j}\right|^{\alpha_{j}} \prod_{k=1}^{N}\left|w-\omega_{k}\right|^{-\beta_{k}}\right) .
$$

This function has negative logarithmic poles at $w_{j}, j=1, \cdots, n$, and positive logarithmic poles at $\omega_{k}, k=1, \cdots, N$, is harmonic elsewhere on the $w$-sphere because of the condition $\sum_{j=1}^{n} \alpha_{j}=\sum_{k=1}^{N} \beta_{k}$. The orthogonal trajectories of its level curves which have a limiting end point at $w_{j}, j=1, \cdots, n$, tend in the other sense to one of the $\omega_{k}, k=1, \cdots, N$, apart from possibly a finite number which tend to critical points of $W(w)$. An analogous remark applies to those orthogonal trajectories which have a limiting end point at $\omega_{k}, k=1, \cdots, N$.

We may assume that $f^{\prime}\left(z_{j}\right), j=1, \cdots, n, F^{\prime}\left(\zeta_{k}\right), k=1, \cdots, N$, are all nonzero since otherwise our inequality (15) is trivially satisfied. Likewise we may assume that $w_{j} \neq w_{l}, j \neq l, \omega_{k} \neq \omega_{m}, k \neq m$. Now we take $t$ sufficiently large that $U_{t}$ consists of $n$ Jordan curves $\gamma_{j}(t), j=1, \cdots, n$, respectively enclosing $z_{j}, j=1, \cdots, n$, that $f\left(\gamma_{j}(t)\right), j=1, \cdots, n$, is a Jordan curve bounding a domain starlike with 
respect to $w_{j}$ for the orthogonal trajectories of the level curves of $W$ and that for any value $-\tau$ of $W(w)$ on $\bigcup_{j=1}^{n} f\left(\gamma_{j}(t)\right)$ the corresponding level set, $W(w)=-\tau$, consists of $n$ Jordan curves $\gamma_{j}^{\prime}(\tau), j=1, \cdots, n$, respectively enclosing $w_{j}, j=1, \cdots, n$. Further we take $s$ sufficiently large that $V_{s}$ consists of $N$ Jordan curves $\lambda_{k}(s)$ $k=1, \cdots, N$, respectively enclosing $\zeta_{k}, k=1, \cdots, N$, that $F\left(\lambda_{k}(s)\right), k=1, \cdots, N$, is a Jordan curve bounding a domain starlike with respect to $\omega_{k}$ for the orthogonal trajectories of the level curves of $W$ and that for any value $\sigma$ of $W(w)$ on $\bigcup_{k=1}^{N} F\left(\lambda_{k}(s)\right)$ the corresponding level set, $W(w)=\sigma$, consists of $N$ Jordan curves $\lambda_{k}^{\prime}(\sigma)$, $k=1, \cdots, N$, respectively enclosing $\omega_{k}, k=1, \cdots, N$.

The image of $|z|<1$ under $w=f(z)$ is now regarded as a Riemann domain which we denote by $\Re$. The image $f\left(\gamma_{j}(t)\right), j=1, \cdots, n$, lies in a simple sheet of $\Re$ containing the image of $z_{j}$. Through each point of $f\left(\gamma_{j}(t)\right)$ we draw a covering of the orthogonal trajectory to the level curves of $W(w)$ through its projection. This is continued on $\Re$ in the sense away from $w_{j}$ as far as possible without the orthogonal trajectory meeting a critical point of $W$ (which can happen at most a finite number of times) and without the covering meeting a branch point of $\mathfrak{R}$ (the latter occurs in at most countably many cases). These curves sweep out a subset $\Sigma_{j}$ of $\Re$ which lies smoothly above the $w$-plane. At all points of $\Sigma_{j}$ we can use $w$ as a local uniformizing parameter on $\Re$ and denote by $g(w)|d w|$ the metric obtained by projecting $\left(2 \pi \sum_{j=1}^{n} \alpha_{j}\right)^{-1}|\operatorname{grad} W||d w|$ up into $\Re$. We can carry this metric back from $\bigcup_{j=1}^{n} \Sigma_{j}$ to its inverse image in $|z|<1$ by the relation

$$
\rho^{\prime}(z)=g(f(z))\left|f^{\prime}(z)\right| .
$$

The metric $\rho(z)|d z|$ obtained by setting

$$
\begin{array}{ll}
\rho(z)=\rho^{\prime}(z) & \text { where latter is defined, } \\
\rho(z)=0 & \text { elsewhere in } D_{t}
\end{array}
$$

is seen at once to be admissible in the module problem defining $M_{t}$.

By an exactly parallel construction on the Riemann domain $\subseteq$ which is the image of $|\zeta|>1$ under the mapping $w=F(\zeta)$ we obtain smooth covering subsets $\Xi_{k}, k=1, \cdots, N$, of $\subseteq$ corresponding to a suitably chosen value $s$ and a metric $\sigma(\zeta)|d \zeta|$ admissible in the module problem defining $N_{s}$.

Let

$$
\tau_{j}=-\min W \text { on } f\left(\gamma_{j}(t)\right), \quad j=1, \cdots, n,
$$

and

$$
\sigma_{k}=\max W \text { on } F\left(\lambda_{k}(s)\right), \quad k=1, \cdots, N .
$$

The projections into the $w$-sphere of $\Sigma_{j}, j=1, \cdots, n$, and $\Xi_{k}, k=1, \cdots, N$, are all disjoint and their union 


$$
\bigcup_{j=1}^{n} \Sigma_{j} \cup \bigcup_{k=1}^{N} \Xi_{k}
$$

is contained in the domain $\left(5\right.$ bounded by the Jordan curves $\gamma_{j}^{\prime}\left(\tau_{j}\right), j=1, \cdots, n$, and $\lambda_{k}^{\prime}\left(\sigma_{k}\right), k=1, \cdots, N$,

We readily deduce

$$
\begin{aligned}
\tau_{j}= & t-\alpha_{j} \log \left|f^{\prime}\left(z_{j}\right)\right|-\log \left(\prod_{l \neq j}\left|w_{j}-w_{l}\right|^{\alpha_{l}} \prod_{k=1}^{N}\left|w_{j}-\omega_{k}\right|^{-\beta_{k}}\right) \\
& +\log \left[\left(1-\left|z_{j}\right|^{2}\right)^{-\alpha_{j}} \prod_{l \neq j}\left|P\left(z_{j}, z_{l}\right)\right|^{\alpha_{l}}\right]+o(1)
\end{aligned}
$$

and

$$
\begin{aligned}
\sigma_{k}= & s-\beta_{k} \log \left|F^{\prime}\left(\zeta_{k}\right)\right|-\log \left(\prod_{j=1}^{n}\left|\omega_{k}-w_{j}\right|^{-\alpha} \prod_{m \neq k}\left|\omega_{k}-\omega_{m}\right|^{\beta_{m}}\right) \\
& +\log \left[\left(1-\left|\zeta_{k}\right|^{2}\right)^{-\beta_{k}} \prod_{m \neq k}\left|P\left(\zeta_{k}, \zeta_{m}\right)\right|^{\beta_{m}}\right]+o(1) .
\end{aligned}
$$

Further we see at once that in the metric $|\operatorname{grad} W||d w|$ the domain $(5$ has area

$$
2 \pi \sum_{j=1}^{n} \alpha_{j} \tau_{j}+2 \pi \sum_{k=1}^{N} \beta_{k} \sigma_{k} .
$$

On the other hand $\left(2 \pi \sum_{j=1}^{n} \alpha_{j}\right)^{-2}$ times this quantity is an upper bound for $M_{t}+N_{s}$. Employing the expressions (16) and (17) we thus have

$$
\begin{aligned}
\frac{1}{2 \pi}\left(\sum_{j=1}^{n} \alpha_{j}\right)^{-2}\left\{\left(\sum_{j=1}^{n} \alpha_{j}\right) t-\sum_{j=1}^{n} \alpha_{j}^{2} \log \left|f^{\prime}\left(z_{j}\right)\right|\right. \\
\quad-\sum_{j=1}^{n} \alpha_{j} \log \left(\prod_{l \neq j}\left|w_{j}-w_{l}\right|^{\alpha_{l}} \prod_{k=1}^{N}\left|w_{j}-\omega_{k}\right|^{-\beta_{k}}\right) \\
+\sum_{j=1}^{n} \alpha_{j} \log \left[\left(1-\left|z_{j}\right|^{2}\right)^{-\alpha_{j}} \prod_{l \neq j} P\left(z_{j}, z_{l}\right)^{\alpha_{l}}\right] \\
+\left(\sum_{k=1}^{N} \beta_{k}\right) s-\sum_{k=1}^{N} \beta_{k}^{2} \log \left|F^{\prime}\left(\zeta_{k}\right)\right| \\
-\sum_{k=1}^{N} \beta_{k} \log \left(\prod_{j=1}^{n}\left|\omega_{k}-w_{j}\right|^{-\alpha_{j}} \prod_{m \neq k}\left|\omega_{k}-\omega_{m}\right|^{\beta_{m}}\right) \\
\left.\quad+\sum_{k=1}^{N} \beta_{k} \log \left[\left(1-\left|\zeta_{k}\right|^{2}\right)^{-\beta_{k}} \prod_{m \neq k}\left|P\left(\zeta_{k}, \zeta_{m}\right)\right|^{\beta_{m}}\right]+o(1)\right\} \\
\geqq\left(2 \pi \sum_{j=1}^{n} \alpha_{j}\right)^{-1} t+\left(2 \pi \sum_{k=1}^{N} \beta_{k}\right)^{-1} s .
\end{aligned}
$$

Recalling that $\sum_{j=1}^{n} \alpha_{j}=\sum_{k=1}^{N} \beta_{k}$, we can cancel the terms in $t$ and $s$ and then take the limit as these quantities tend to infinity. We obtain 


$$
\begin{aligned}
& -\sum_{j=1}^{n} \alpha_{j}^{2} \log \left|f^{\prime}\left(z_{j}\right)\right|-\sum_{j=1}^{n} \alpha_{j} \log \left(\prod_{l \neq j}\left|w_{j}-w_{l}\right|^{\alpha_{l}} \prod_{k=1}^{N}\left|w_{j}-\omega_{k}\right|^{-\beta_{k}}\right) \\
& +\sum_{j=1}^{n} \alpha_{j} \log \left[\left(1-\left|z_{j}\right|^{2}\right)^{-\alpha_{l}} \prod_{l \neq j} P\left(z_{j}, z_{l}\right)^{\alpha_{l}}\right\rfloor \\
& -\sum_{k=1}^{N} \beta_{k}^{2} \log \left|F^{\prime}\left(\zeta_{k}\right)\right|-\sum_{k=1}^{N} \beta_{k} \log \left(\prod_{j=1}^{n}\left|\omega_{k}-w_{j}\right|^{-\alpha_{j}} \prod_{m \neq k}\left|\omega_{k}-\omega_{m}\right|^{\beta_{m}}\right) \\
& +\sum_{k=1}^{N} \beta_{k} \log \left[\left(1-\left|\zeta_{k}\right|^{2}\right)^{-\beta_{k}} \prod_{m \neq k}\left|P\left(\zeta_{k}, \zeta_{m}\right)\right|^{\beta_{m}}\right] \geqq 0 \text {. }
\end{aligned}
$$

Exponentiating, we obtain the inequality (15).

Theorem 2 has variants obtained by allowing some $f\left(z_{j}\right), j=1, \cdots n, \zeta_{k}, F\left(\zeta_{k}\right)$, $k=1, \cdots, N$, to be the point at infinity. Instead of stating these in the general case we confine ourselves to the following situation.

THEOREM 2'. Let $f(z)$ be regular in $|z|<1$ and satisfy $f(0)=0$. Let $F(\zeta)$ be meromorphic in $|\zeta|>1$ and have Laurent expansion about the point at infinity

$$
F(\zeta)=c \zeta+c_{0}+c_{1} \zeta^{-1}+\cdots .
$$

Let the images of $|z|<1$ by $w=f(z)$ and of $|\zeta|>1$ by $w=F(\zeta)$ be nonoverlapping. Let $\alpha_{1}, \alpha_{2}, \beta_{1}, \beta_{2}$ be positive constants such that $\alpha_{1}+\alpha_{2}=\beta_{1}+\beta_{2}$. Then for $|z|<1,|\zeta|>1$,

$$
\begin{aligned}
\left|f^{\prime}(0)\right|^{\alpha_{1}^{2}}\left|f^{\prime}(z)\right|^{\alpha_{2}^{2}}\left|F^{\prime}(\zeta)\right|^{\beta_{2}^{2}}|c|^{-\beta_{1}^{2}}|f(z)|^{2 \alpha_{1} \alpha_{2}}|F(\zeta)|^{-2 \alpha_{1} \beta_{2}}|f(z)-F(\zeta)|^{-2 \alpha_{2} \beta_{2}} \\
\leqq|z|^{2 \alpha_{1} \alpha_{2}}|\zeta|^{-2 \beta_{1} \beta_{2}}\left(1-|z|^{2}\right)^{-\alpha_{2}^{2}}\left(|\zeta|^{2}-1\right)^{-\beta_{2}^{2}}
\end{aligned}
$$

This result is obtained from (15) by a limiting process or can be obtained by direct application of the method of proof of Theorem 2 in this situation. It generalizes a result of Alenitsyn [1] who required that $f$ and $F$ be univalent and that $\alpha_{1}=\alpha_{2}=\beta_{1}=\beta_{2}=1$.

It seems worthwhile to emphasize again the essential role played in the proof of Theorem 2 by the structure of the orthogonal trajectories of the level curves of $W(w)$, especially since Pfluger and Hersch [4] seem to have missed this point in the paper where these methods were used for the first time [5]. Rather than entailing "restrictive hypotheses," it is the structure of such curves which enables one to relate the module of a family of image curves to the module of a curve family with similar topological situation.

5. Corollary 11. Let $f(z) \in C$. Let $\alpha_{j}, j=1, \cdots, n, \beta_{k}, k=1, \cdots, N$ be positive constants such that $\sum_{j=1}^{n} \alpha_{j}=\sum_{k=1}^{N} \beta_{k}$. Let $z_{j}, j=1, \cdots, n, Z_{k}, k=1, \cdots, N$, be points in $|z|<1$. Then 


$$
\prod_{j=1}^{n}\left|f^{\prime}\left(z_{j}\right)\right|^{\alpha_{j}^{2}} \prod_{k=1}^{N}\left|f^{\prime}\left(Z_{k}\right)\right|^{\beta_{k}^{2}} \prod_{j \neq l ; j, l}\left|f\left(z_{j}\right)-f\left(z_{l}\right)\right|^{2 \alpha_{j} \alpha_{l}}
$$

$$
\begin{gathered}
\cdot \prod_{k \neq m ; k, m}\left|f\left(Z_{k}\right)-f\left(Z_{m}\right)\right|^{2 \beta_{k} \beta_{m}} \prod_{j=1}^{n} \prod_{k=1}^{N}\left|1-f\left(z_{j}\right) f\left(Z_{k}\right)\right|^{-2 \alpha_{j} \beta_{k}} \\
\leqq \prod_{j \neq l ; j, l}\left|P\left(z_{j}, z_{l}\right)\right|^{2 \alpha_{j} \alpha_{l}} \prod_{k \neq m ; k, m}\left|P\left(Z_{k}, Z_{m}\right)\right|^{2 \beta_{k} \beta_{m}} \prod_{j=1}^{n}\left(1-\left|z_{j}\right|^{2}\right)^{-\alpha_{j}^{2}} \prod_{k=1}^{N}\left(1-\left|Z_{k}\right|^{2}\right)^{-\beta_{k}^{2} \cdot}
\end{gathered}
$$

This is obtained by setting in $(15), F(\zeta)=\left(f\left(\zeta^{-1}\right)\right)^{-1}, \zeta_{k}=Z_{k}^{-1}, k=1, \cdots, N$. This presumes a priori that $Z_{k}, f\left(Z_{k}\right), k=1, \cdots, N$ is not zero. However this condition can evidently be dropped.

Corollary 12. Let $f(z) \in C$. Let $\alpha_{1}, \alpha_{2}, \beta_{1}, \beta_{2}$ be positive constants such that $\alpha_{1}+\alpha_{2}=\beta_{1}+\beta_{2}$. Let $|z|<1,|Z|<1$. Then

$$
\begin{aligned}
& \left|f^{\prime}(0)\right|^{\alpha_{1}^{2}+\beta_{1}^{2}}\left|f^{\prime}(z)\right|^{\alpha_{2}^{2}}\left|f^{\prime}(Z)\right|^{\beta_{2}^{2}}|f(z)|^{2 \alpha_{1} \alpha_{2}}|f(Z)|^{2 \beta_{1} \beta_{2}}|1-f(z) F(Z)|^{-2 \alpha_{2} \beta_{2}} \\
& \leqq|z|^{2 \alpha_{1} \alpha_{2}}|Z|^{2 \beta_{1} \beta_{2}}\left(1-|z|^{2}\right)^{-\alpha_{2}^{2}}\left(1-|Z|^{2}\right)^{-\beta_{2}^{2}}
\end{aligned}
$$

This is obtained by setting in $(18), F(\zeta)=\left(f\left(\zeta^{-1}\right)\right)^{-1}, \zeta=Z^{-1}$. This presumes a priori that $Z \neq 0$ but this condition is evidently superfluous. It generalizes a result of Alenitsyn [1] to which it reduces by taking $\alpha_{1}=\alpha_{2}=\beta_{1}=\beta_{2}=2^{-1 / 2}, z=Z$.

Corollary 13. Let $f(z) \in C$. Let $|z|<1,|z|<1$. Then

$$
\left|f^{\prime}(z) f^{\prime}(Z)\right||1-f(z) f(Z)|^{-2} \leqq\left(1-|z|^{2}\right)^{-1}\left(1-|Z|^{2}\right)^{-1} \text {. }
$$

This is obtained by setting in (19), $\alpha_{1}=\beta_{1}=1$. It generalizes a result of Gelfer [2] to which it reduces by taking $Z=z$ and one of Alenitsyn [1] to which it reduces by taking $Z=0$.

Corollary 14. Let $f(z) \in K$. Let $\alpha_{j}, j=1, \cdots, n, \beta_{k}, k=1, \cdots, N$, be positive real constants such that $\sum_{j=1}^{n} \alpha_{j}=\sum_{k=1}^{N} \beta_{k}$. Let $z_{j}, j=1, \cdots, n, Z_{k}, k=1, \cdots, N$, be points in $|z|<1$. Then

$$
\begin{aligned}
& \prod_{j=1}^{n}\left|f^{\prime}\left(z_{j}\right)\right|^{\alpha_{j}^{2}} \prod_{k=1}^{N}\left|f^{\prime}\left(Z_{k}\right)\right|^{\beta_{k}^{2}} \prod_{j \neq l ; j, l}\left|f\left(z_{j}\right)-f\left(z_{l}\right)\right|^{2 \alpha_{j} \alpha_{l}} \prod_{k \neq m ; k, m}\left|f\left(Z_{k}\right)-f\left(Z_{m}\right)\right|^{2 \beta_{k} \beta} \\
& \quad \prod_{j=1}^{n} \prod_{k=1}^{N}\left|1+f\left(z_{j}\right) f\left(Z_{k}\right)\right|^{-2 \alpha_{j} \beta_{k}} \\
& \leqq \prod_{j \neq l ; j, l}\left|P\left(z_{j}, z_{l}\right)\right|^{2 \alpha_{j} \alpha_{l}} \prod_{k \neq m ; k, m}\left|P\left(Z_{k}, Z_{m}\right)\right|^{2 \beta_{k} \beta_{m}} \prod_{j=1}^{n}\left(1-\left|z_{j}\right|^{2}\right)^{-\alpha_{j}^{2}} \prod_{k=1}^{N}\left(1-\left|Z_{k}\right|^{2}\right)^{-\beta_{k}^{2}}
\end{aligned}
$$

This is obtained by setting in $(15), F(\zeta)=-\left(\bar{f}\left(\zeta^{-1}\right)\right)^{-1}, \zeta_{k}^{-1}=Z_{k}, k=1, \cdots, N$. This presupposes a priori that $Z_{k}, f\left(Z_{k}\right), k=1, \cdots, N$, is not zero. This condition is evidently superfluous. 
Corollary 15. Let $f(z) \in K$. Let $\alpha_{1}, \alpha_{2}, \beta_{1}, \beta_{2}$ be positive constants such that $\alpha_{1}+\alpha_{2}=\beta_{1}+\beta_{2}$. Let $|z|<1,|Z|<1$. Then

$$
\begin{aligned}
\left|f^{\prime}(0)\right|^{\alpha_{1}^{2}+\beta_{1}^{2}}\left|f^{\prime}(z)\right|^{\alpha_{2}^{2}}\left|f^{\prime}(Z)\right|^{\beta_{2}^{2}} \mid & \left.f(z)\right|^{2 \alpha_{1} \alpha_{2}}|f(Z)|^{2 \beta_{1} \beta_{2}}|1+f(z) f(Z)|^{-2 \alpha_{2} \beta_{2}} \\
& \leqq|z|^{2 \alpha_{1} \alpha_{2}}|Z|^{2 \beta_{1} \beta_{2}}\left(1-|z|^{2}\right)^{-\alpha_{2}^{2}}\left(1-|Z|^{2}\right)^{-\beta_{2}^{2}} .
\end{aligned}
$$

This is obtained by setting in $(18), F(\zeta)=-\left(\bar{f}\left(\zeta^{-1}\right)\right)^{-1}, Z=\zeta^{-1}$.

There are of course many other special cases of the preceding results which have considerable interest. Also they can be combined with the earlier results in various manners.

6. We will now give an application of the General Coefficient Theorem to the class $K^{*}$, choosing the particular problem of maximizing $\left|A_{2}\right|$ in the expansion

$$
f(z)=A_{1} z+A_{2} z^{2}+\cdots
$$

when $\left|A_{1}\right|$ is given. We begin with the construction of the functions which play the extremal role. Let $\Lambda(t), t \geqq 0$, denote the domain in the $\zeta$-plane $(\zeta=\xi+i \eta)$ bounded by the half-lines

$$
\begin{array}{ll}
\eta=t, & \xi \leqq-1, \\
\xi=-1, & \eta \leqq t, \\
\eta=-t, & \xi \geqq 1, \\
\xi=1, & \eta \geqq-t .
\end{array}
$$

We denote the points $-1+i t, 1-i$ by $B, D$ and the boundary points of $\Lambda(t)$ at infinity by $A, C$ so that $A, B, C, D$ lie on the boundary of $\Lambda(t)$ in natural cyclic order. We map $\Lambda(t)$ conformally onto the left-hand half-plane $\mathfrak{R} w<0$ so that $A$ corresponds to $w=0, D$ to $w=\infty$. Since the mapping $\zeta \rightarrow-\zeta$ of $\Lambda(t)$ onto itself corresponds to a linear transformation of the half-plane onto itself interchanging the origin and the point at infinity we may further normalize the mapping so that $\zeta=0$ corresponds to $w=-1$. Then $B$ and $D$ correspond respectively to points $i a,-i / a$ for $a>0$.

If we extend $\zeta$ as a (non-single-valued) function of $w$ to the whole $w$-plane by reflection in various segments of the imaginary axis we see that $d \zeta^{2}$ is a quadratic differential on the $w$-sphere with triple poles at $0, \infty$ and simple zeros at $i a,-i a^{-1}$. Thus we can write

$$
d \zeta^{2}=q i \frac{(w-i a)\left(w+i a^{-1}\right)}{w^{3}} d w^{2}
$$

for a suitable positive constant $q$.

Let us map the semicircle $|z|<1, \Re z<0$ onto the portion of $\Lambda(t)$ in the upper half-plane so that the origin corresponds to $A$, the point $z=i$ to $\zeta=-1$ 
and the point $z=-i$ to $\zeta=1$. Combining this with the above mapping into the $w$-plane and extending by reflection across the segment joining $i$ and $-i$ in the $z$-plane and its corresponding segment in the $w$-plane we obtain a function regular and univalent for $|z|<1$ which we denote by $f(z, t)$. We denote the image of $|z|<1$ under the mapping $w=f(z, t)$ by $D(t)$. The latter is bounded by a trajectory of $d \zeta^{2}$ for $t>0$ and by two trajectories of $d \zeta^{2}$ having common end points at $i a,-i a^{-1}$ for $t=0$. Clearly $f^{\prime}(0, t)>0$.

Let $a^{*}$ be the value of $a$ for $t=0$. Let $D(-s)$ be the subdomain of $D(0)$ obtained by slitting it along the segment $\mathfrak{R} w=0, s \leqq \mathfrak{I} w \leqq a^{*}$, where $0<s<a^{*}$. Let $f(z,-s)$ be the function mapping $|z|<1$ conformally onto $D(-s)$ so that $f(0,-s)=0, f^{\prime}(0,-s)>0$.

In this way $f(z, t)$ is defined for $t>-a^{*}$ so as to depend continuously on the parameter $t$ in the evident manner. Let it have expansion about the origin

$$
f(z, t)=\lambda(t) z+i \kappa(t) z^{2}+\cdots .
$$

We see that $\lambda(t)>0$ and $\kappa(t)$ is real. They are continuous functions of $t$.

LEMMA 1. Let $f(z) \in K^{*}$ with $f^{\prime}(0)=\lambda, \lambda>0$, and let $f(z, t)$ be such that $\lambda(t)=\lambda$. Let $f(z)$ have expansion about the origin

$$
f(z)=\lambda z+A_{2} z^{2}+\cdots .
$$

Then $\left|A_{2}\right| \leqq \kappa(t)$ with $A_{2}=i \kappa(t) e^{-i \theta}$ occurring only for $f(z)=e^{i \theta} f\left(e^{-i \theta} z, t\right), \theta$ real.

Let $\Phi(w, t, \theta)$ be the function inverse to the function $e^{i \theta} f\left(e^{-i \theta} z, t\right)$ which we denote by $f(z, t, \theta)$. It is defined in the domain $e^{i \theta} D(t)$ which we denote by $D(t, \theta)$. Let $\Psi(w, t, \theta)$ be the function inverse to the function $-\left(\tilde{f}\left(\bar{z}^{-1}, t, \theta\right)\right)^{-1}$. It is defined in the domain $D^{\prime}(t, \theta)$ obtained from $D(t, \theta)$ by the transformation $w \rightarrow-\bar{w}^{-1}$.

We apply the General Coefficient Theorem [9] with $\mathfrak{R}$ the $w$-sphere,

$$
\begin{aligned}
Q(w) d w^{2} & =i e^{-i \theta} \frac{\left(w-i e^{i \theta} a\right)\left(w+i e^{i \theta} a^{-1}\right)}{w^{3}} d w^{2}, \quad t>0, \\
& =i e^{-i \theta} \frac{\left(w-i e^{i \theta} a^{*}\right)\left(w+i e^{i \theta}\left(a^{*}\right)^{-1}\right)}{w^{3}} d w^{2}, \quad t \leqq 0,
\end{aligned}
$$

the admissible family of domains consisting of $\left\{D(t, \theta), D^{\prime}(t, \theta)\right\}$. The admissible family of functions consists of $\left\{f(\Phi(w, t, \theta)),-\left(\bar{f}\left((\bar{\Psi}(w, t, \theta))^{-1}\right)\right)^{-1}\right\}$.

The quadratic differential has poles $P_{1}, P_{2}$ of third order respectively at the origin and the point at infinity. The corresponding coefficients are

$$
\begin{aligned}
& \alpha^{(1)}=i e^{i \theta}, \quad a_{0}^{(1)}=\left(i e^{-i \theta} \kappa(t)-A_{2}\right) \lambda^{-2}, \\
& \alpha^{(2)}=i e^{-i \theta}, \quad a_{0}^{(2)}=\left(i e^{i \theta} \kappa(t)+A_{2}\right) \lambda^{-2} .
\end{aligned}
$$

The General Coefficient Theorem then gives 


$$
\Re\left\{i e^{i \theta}\left(i e^{-i \theta} \kappa(t)-A_{2}\right) \lambda^{-2}+i e^{-i \theta}\left(\bar{A}_{2}+i e^{i \theta} \kappa(t)\right) \lambda^{-2}\right\} \leqq 0
$$

or

or

$$
\mathfrak{R}\left\{-i e^{i \theta} A_{2}+i e^{-i \theta} \bar{A}_{2}\right\} \leqq 2 \kappa(t)
$$

$$
\Re\left\{-i e^{i \theta} A_{2}\right\} \leqq \kappa(t) .
$$

Suitable choice of $\theta$ gives

$$
\left|A_{2}\right| \leqq \kappa(t)
$$

The equality statement follows from equality condition (i) in the General Coefficient Theorem [9].

It is evident that the functions $f(z, t)$ are all distinct. Since they all belong to $K^{*}$ it follows from Lemma 1 that $\lambda(t)$ is a monotone function of $t$. As $t$ approaches $-a^{*}$ clearly $\lambda(t)$ tends to zero. Thus $\lambda(t)$ increases monotonically with $t$. Since $K^{*}$ is a normal family, from any sequence $\left\{f\left(z, t_{n}\right)\right\}_{1}^{\infty}$ with $\lim _{n \rightarrow \infty} t_{n}=\infty$ we can extract a convergent subsequence. As $t \rightarrow \infty, D(t)$ converges to the strip $-1<\mathfrak{J}<1$, so the corresponding mapping will converge to $w=-\exp \left(\frac{1}{2} \pi i \zeta\right)$. The same is true for the mapping corresponding to the $z$-plane. Thus the limiting function of the above subsequence must be $z$. In particular as $t$ tends to infinity $\lambda(t)$ tends to 1 .

Given $\lambda, 0<\lambda<1$, there is a unique function $f(z, t), t>-a^{*}$ with $f^{\prime}(0, t)=\lambda$. We denote this function by $E(z, \lambda)$ and its expansion about the origin by

$$
E(z, \lambda)=\lambda z+i v(\lambda) z^{2}+\cdots .
$$

We include the case $\lambda=1$ by setting $E(z, 1) \equiv z$. The result of Lemma 1 may be rephrased as

THEOREM 3. Let $0<\lambda \leqq 1$ and let $f(z) \in K^{*}$ with expansion about the origin

$$
f(z)=\lambda z+A_{2} z^{2}+\cdots .
$$

Then $\left|A_{2}\right| \leqq v(\lambda)$ with $A_{2}=i e^{-i \theta} v(\lambda)$ occurring only for $f(z)=e^{i \theta} E\left(e^{-i \theta} z, \lambda\right), \theta$ real.

The case $\lambda=1$ is trivial.

7. We can apply the General Coefficient Theorem also to the class $C^{*}$ but in this case the admissible family of functions would have to be $\{f(\Phi(w, t, \theta))$, $\left.\left(f\left(-(\Psi(w, t, \theta))^{-1}\right)\right)^{-1}\right\}$ and the preceding argument would lead to the inequality

$$
\Re\left\{-i \cos \theta A_{2}\right\} \leqq \kappa(t)
$$

instead of (23) and thus would not provide a bound for $\left|A_{2}\right|$. However by utilizing our previous results on the bound for $\left|f\left(r e^{i \theta}\right)\right|$ when $\left|A_{1}\right|$ is given we are able to prove that $\left|A_{2}\right| \leqq v(\lambda)$ for $\left|A_{1}\right|=\lambda$ in this case also. We will carry over the notations of the paper [6] without necessarily repeating the definitions. We consider 
the function $-F(-z ; r, \lambda)$ in order to correspond to our present normalization. This function satisfies the differential equation

$$
\frac{(w+i l)\left(w-i l^{-1}\right)}{w^{2}(w+i m)\left(w-i m^{-1}\right)}\left(\frac{d w}{d z}\right)^{2}=\frac{(z+i p)\left(z+i p^{-1}\right)}{z^{2}(z+i r)\left(z+i r^{-1}\right)}
$$

in which $0<r<1, r \geqq p \geqq-1, m>0$ where $-i m$ is the image of $-i r$ under the mapping $w=-F(-z ; r, \lambda)$ and where $-i l$ is either the image of $-i p$ under this mapping (when $p>-1$ ) or lies in the boundary of the image of $|z|<1$ (when $p=-1$ ). We know that if $f(z) \in C^{*}$ with $\left|f^{\prime}(0)\right|=\lambda$ then $\left|f\left(r e^{i \theta}\right)\right| \leqq m$. Moreover as $p$ varies from $r$ to -1 the corresponding $\lambda$ decreases monotonically. In particular when $p=0$ we get the overall maximum of $\left|f\left(r e^{i \theta}\right)\right|$ the corresponding function $F(z ; r, \lambda)$ being $\left(1-r^{2}\right)^{1 / 2} z(1+i r z)^{-1}$ where $\lambda=\left(1-r^{2}\right)^{1 / 2}$. Thus for given $\lambda, 0<\lambda<1$, by choosing $r$ small enough we may assume that $-1 \leqq p<0$. Then the corresponding value $l$ is less than zero. Now from the functions $-F(-z ; r, \lambda)$ as $r$ approaches zero we can extract a convergent subsequence and the limit function is evidently in $C^{*}$ with derivative $\lambda$ at $z=0$. By choosing a subsubsequence if necessary we may assume that the associated values $l$ also converge to a value $l^{*}$ a priori possibly 0 or $-\infty$. The latter are excluded if the corresponding values of $p$ are -1 . If the corresponding values of $p$ are $>-1$ they must also converge and $l^{*}$ cannot be $-\infty$. It can be zero only if the limit for $p$ is zero. Otherwise we may denote either -1 or this limiting value by $p^{*}$.

Recalling that the coefficients in the explansion about the origin of a function in $C$ are uniformly bounded by 1 in absolute value [11] we have

$$
|m-\lambda r| \leqq r^{2}(1-r)^{-1} \text {. }
$$

Thus as $r \rightarrow 0$ we have $m / r \rightarrow \lambda$. If we had $l \rightarrow 0$ we would have also $l / p \rightarrow \lambda$. Thus the limiting function, denoted by $h(z)$, satisfies the differential equation

if $l^{*} \neq 0$ or

$$
i \frac{\left(w+i l^{*}\right)\left(w-i\left(l^{*}\right)^{-1}\right)}{w^{3}}\left(\frac{d w}{d z}\right)^{2}=i \frac{\left(z+i p^{*}\right)\left(z+i\left(p^{*}\right)^{-1}\right)}{z^{3}}
$$

$$
-\frac{1}{w^{2}}\left(\frac{d w}{d z}\right)^{2}=-\frac{1}{z^{2}}
$$

if $l^{*}=0$. In the latter case the limiting function would have to be $\lambda z$. However \pm 1 are on the boundary of the image of $|z|<1$ under the mapping $w=-F(-z ; r, \lambda)$ and it follows by domain convergence that \pm 1 must be on the boundary of the image of $|z|<1$ under the mapping $w=h(z)$. This would be impossible if $l^{*}=0$. Hence $l^{*} \neq 0$ and $w=h(z)$ maps $|z|<1$ onto an admissible domain with respect to the quadratic differential

$$
i \frac{\left(w+i l^{*}\right)\left(w-i / l^{*}\right)}{w^{3}} d w^{2} .
$$


Once again \pm 1 are boundary points of the image and thus $h(z)$ is exactly that function $f(z, t)$ introduced in $\S 6$ for which $f^{\prime}(0, t)=\lambda$.

Now suppose there were a function $f(z) \in C^{*}$ with expansion

$$
f(z)=\lambda z+A_{2} z^{2}+\cdots
$$

and $\left|A_{2}\right|>v(\lambda)$. Then for suitable real $\theta$

while

$$
\left|f\left(r e^{i \theta}\right)\right| \geqq \lambda r+\left|A_{2}\right| r^{2}-r^{3}(1-r)^{-1},
$$

$$
|F(i r ; r, \lambda)| \leqq \lambda r+(v(\lambda)+o(1)) r^{2}+r^{3}(1-r) .
$$

By choosing $r$ sufficiently small we obtain an evident contradiction. Thus we may state

THEOREM 4. Let $0<\lambda \leqq 1$ and let $f(z) \in C^{*}$ with expansion about the origin

$$
f(z)=\lambda z+A_{2} z^{2}+\cdots .
$$

Then $\left|A_{2}\right| \leqq v(\lambda)$.

The case $\lambda=1$ is trivial. A proof of this kind does not normally give an equality statement.

8. There have been several approaches to defining families of functions in analogy to the Bieberbach-Eilenberg functions. Of course there is Shah's definition, indicated above. Further Goodman [3], concentrating on the fact that the ransformations $w \rightarrow w, w \rightarrow w^{-1}$ form a group of linear transformations on the $w$-sphere, considered families of functions conditioned by their behaviour relative to more general groups of linear transformations. Other extensions in similar directions could also be made. However it appears that perhaps the most evident extension has not been considered. Indeed, given any closed set $S$ of points in the sphere not containing the origin or the point at infinity, we can consider the family which we may denote by $C\{S\}$ consisting of functions $f(z)$ regular for $|z|<1$, with $f(0)=0$, which satisfy the condition

$$
f\left(z_{1}\right) f\left(z_{2}\right) \notin S, \quad\left|z_{1}\right|<1,\left|z_{2}\right|<1 .
$$

Similarly we can define the family $K\{S\}$ for which the condition (24) is replaced by

$$
f\left(z_{1}\right) \tilde{f}\left(z_{2}\right) \notin S, \quad\left|z_{1}\right|<1,\left|z_{2}\right|<1 .
$$

Any such family is normal and thus it is significant to study the standard extremal problems for them. We denote the corresponding families of univalent functions by $C^{*}\{S\}, K^{*}\{S\}$ respectively.

The simplest case would be where $S$ consists of a single point, say $a$. We will use for the corresponding families the simplified notations $C\{a\}, K\{a\}$. The 
family $C\{a\}$ differs from $C$ only by a nonessential normalization. Indeed if $f(z) \in C\{a\}$ the function $a^{-1 / 2} f(z) \in C$ (for either choice of root). However the families $K\{a\}$ display interesting new phenomena. Once again by normalization it is sufficient to confine our attention to the families $K\left\{e^{i \theta}\right\}, \theta$ real. $K\{-1\}$ is the family $K . K\{1\}$ is the family of bounded functions, i.e. those with $|f(z)|<1$ for $|z|<1$. Thus the results of $\S 2$ and $\S 4$ can be applied to obtain numerous results for such functions. The condition that $f \in K\left\{e^{i \theta}\right\}$ can be expressed by saying that the images of $|z|<1$ by $f$ and $e^{i \theta} \bar{f}^{-1}$ do not overlap. Every extremal function we have met for $K$ has the property that the closures of the appropriate nonoverlapping images fill the whole sphere. An analogous situation obtains for $C$. In the general situation, however, something quite new occurs.

THEOREM 5. Let $f \in K\left\{e^{i \theta}\right\}$ where $\theta / \pi$ is irrational. Then the closures of the images of $|z|<1$ by fand $e^{i \theta} \bar{f}^{-1}$ fill the whole sphere only if $|f(z)|<1$ for $|z|<1$.

We note first of all that if $f \in K\left\{e^{i \theta}\right\}$, also $f \in K\left\{e^{-i \theta}\right\}$. Now suppose that the closures of the images of $|z|<1$ by $f$ and $e^{i \theta} \bar{f}^{-1}$ fill the whole sphere while, for $|z|<1, f(z)$ assumes a value $c$ with $|c|>1$. Then $e^{i \theta \tilde{f}^{-1}}$ takes for $|z|<1$ all values in a neighborhood of $e^{i \theta} \bar{c}^{-1}$ and $f$ omits these values. This means that $e^{i \theta} \bar{f}^{-1}$ must omit all values in a neighborhood of $e^{2 i \theta} c$ thus $f$ must take this value. Then $f$ would take for $|z|<1$ all values $e^{2 n i \theta} c, n$ integral. Reduced modulo $2 \pi$ the values $2 n \theta$ lie dense on the interval $[0,2 \pi)$ thus $f$ would take values dense on the circle of radius $|c|>1$. This would contradict the assertion that $e^{i \theta} \tilde{f}^{-1}$ assumes a value of modulus less than 1 .

It is clear that certain simple extremal problem will have as solutions functions $f$ not satisfying the condition $|f(z)|<1$ for $|z|<1$. Then for these functions there are points exterior to both the images of $|z|<1$ by $f$ and $e^{i \theta} \bar{f}^{-1}$. We will not pursue this matter further here.

In case $\theta / \pi$ is rational we have a situation much closer to that for $K$. In particular we have the following result.

THEOREM 6. If $f \in K\{i\}$ then it is subordinate to an odd function in $K^{*}\{i\}$. The odd functions in $K^{*}\{i\}$ coincide with the set of functions $\left(g\left(z^{2}\right)\right)^{1 / 2}$ where $g \in K^{*}$.

Let $D$ be the union of the images of $|z|<1$ by $f$ and $-f$. Since $K\{i\}=K\{-i\}$, $D$ has no point in common with the image of $|z|<1$ by $i \tilde{f}^{-1}$ or $-i \tilde{f}^{-1}$. Let $\Delta$ be the complement of that component of the complement of $D$ which contains the point at infinity. It is evidently symmetric in the origin and does not meet its image under the transformation $w \rightarrow i \bar{w}^{-1}$. Thus a function $F(z)$ mapping $|z|<1$ conformally onto $\Delta$ with $F(0)=0$ is an odd function in $K^{*}\{i\}$. Clearly $f(z)$ is subordinate to $F(z)$. The last statement of the theorem is explicitly verified at once. 
COROllary 16. Let $f \in K\{i\}$. Then for $\theta$ real, $0<r<1$,

$$
\left|f\left(r e^{i \theta}\right)\right| \leqq r\left(1-r^{4}\right)^{-1 / 4} \text {. }
$$

Equality occurs only for the functions $e^{i \phi}\left(1-r^{4}\right)^{1 / 4} z\left(1-r^{2} e^{-2 i \theta} z^{2}\right)^{-1 / 2}, \phi$ real.

This follows from Theorem 6, Corollary 10 and the equality statement for the latter (see [9, Theorem 7.2]).

9. In the paper [6] it was stated that the precise bound for $\left|f\left(r e^{i \theta}\right)\right|, f \in C$, $0<r<1, \theta$ real, $\left|f^{\prime}(0)\right|=c, 0<c \leqq 1$, follows from the corresponding bound for $f \in C^{*}$ by "subordination." It is easily seen that this statement is unjustified as was pointed out by Hayman in his review of that paper (Math. Reviews 16 (1955), 25 ) although to this day I have not seen what the example he gives is supposed to prove. The reason that the subordination argument fails is because, although $f$ is subordinate to an $F \in C^{*}$ we will in general have $\left|F^{\prime}(0)\right|>c$. Oddly enough this very point was treated meticulously in the paper [7] which followed shortly after.

However a considerable part of the above statement is actually true. To see this one must study the behaviour of the quantity $\mathfrak{I} F(i r ; r, c)$ for fixed $r$ and $0<c \leqq 1$. We know that, as a function of $c$, this is continuous, tends to zero as $c \rightarrow 0+$, takes the value $r$ when $c=1$ and is maximal for $c=\left(1-r^{2}\right)^{1 / 2}$. Now we can see that actually it increases monotonically as $c$ goes from $0+$ to $\left(1-r^{2}\right)^{1 / 2}$ and decreases monotonically as $c$ goes from $\left(1-r^{2}\right)^{1 / 2}$ to 1 .

LEMMA 2. For fixed $r, 0<c \leqq 1, \mathfrak{I} F(i r ; r, c)$ increases (strictly) monotonically from 0 to $r\left(1-r^{2}\right)^{-1 / 2}$ as $c$ increases from $0+$ to $\left(1-r^{2}\right)^{1 / 2}$ and decreases (strictly) monotonically from $r\left(1-r^{2}\right)^{-1 / 2}$ to $r$ as $c$ increases from $\left(1-r^{2}\right)^{1 / 2}$ to 1 .

This follows by the same arguments used to prove the extremal proporties in [6] and indeed without the application of any symmetrization.

TheOREM 7. Let $f(z) \in C, 0<r<1,\left|f^{\prime}(0)\right|=c,\left(1-r^{2}\right)^{1 / 2} \leqq c \leqq 1$. Then for $\theta$ real

$$
\left|f\left(r e^{i \theta}\right)\right| \leqq \mathfrak{I} F(i r ; r, c) .
$$

Equality can occur only for the (univalent) functions $\pm F\left(i e^{-i \theta} z ; r, c\right)$.

Indeed $f(z)$ is subordinate to a function $F(z) \in C^{*}$ with $\left|F^{\prime}(0)\right|=c^{\prime} \geqq c$. Now

$$
\max _{z \leqq r}|f(z)| \leqq \max _{z \leqq r}|F(z)| \leqq \Im F\left(i r ; r, c^{\prime}\right) \leqq \Im F(i r ; r, c)
$$

by [ 6 , Theorem 3$]$ and Lemma 2 . The equality statement follows by a consideration of when equality can occur in the above chain of inequalities together with an application of the uniqueness result for symmetrization [8]. The latter result was of course obtained after the appearance of [6].

Naturally the parallel result applies to the family $K$. 
THEOREM 8. Let $f(z) \in K, 0<r<1,\left|f^{\prime}(0)\right|=c,\left(1-r^{2}\right)^{1 / 2} \leqq c \leqq 1$. Then for $\theta$ real

$$
\left|f\left(r e^{i \theta}\right)\right| \leqq \mathfrak{I} F(i r ; r, c) .
$$

Equality can occur only for the (univalent) functions $e^{i \phi} F\left(i e^{-i \theta} z ; r, c\right), \phi$ real.

This is obtained by proving a parallel to [6, Theorem 3] for $f$ univalent (which does not need a symmetrization argument) and then using the same proof as for Theorem 7. Alternatively the proof can be given directly without a subordination argument by using the method of simple coverings as in $\S 4$.

\section{BIBLIOGRAPHY}

1. I. E. Alenitsyn, A contribution to the theory of schlicht functions and BieberbachEilenberg functions, Dokl. Akad. Nauk SSSR. 109 (1956), 247-249. (Russian)

2. S. A. Gelfer, On the class of regular functions not taking on any pair of values $w$ and $-w$, Mat. Sb. (N.S.) 19 (1946), 33-44. (Russian. English summary)

3. A. W. Goodman, Almost bounded functions, Trans. Amer. Math. Soc. 78 (1955), 82-97.

4. J. Hersch and A. Pfluger, Principe de l'augmentation des longueurs extrémales, C. R. Acad. Sci. Paris 237 (1953), 1205-1207.

5. James A. Jenkins, Some results related to extermal length, Contributions to the Theory of Riemann Surfaces, Annals of Mathematics Studies No. 30, pp. 87-94, Princeton Univ. Press, Princeton, N. J., 1953.

6. - On Bieberbach-Eilenberg functions, Trans. Amer. Math. Soc. 76 (1954), 389-396.

7. - On Bieberbach-Eilenberg functions. II, Trans. Amer. Math. Soc. 78 (1955), $510-515$.

8. - Some uniqueness results in the theory of symmetrization, Ann. of Math. (2) 61 (1955), 106-115.

9. - Univalent functions and conformal mapping, Springer-Verlag, Berlin, 1958.

10. - Some area theorems and a special coefficient theorem, Illinois J. Math. 8 (1964), 80-99.

11. N. A. Lebedev and I. M. Milin, On the coefficients of some classes of analytic functions, Mat. Sb. (N.S.) 28 (1951), 359-400. (Russian)

12. N. A. Lebedev, Applications of the area principle in problems for non-overlapping domains, Trudy Mat. Inst. Steklov. 60 (1961), 211-231. (Russian)

13. Tao-Shing Shah, On the moduli of some classes of analytic functions, Acta Math. Sinica 5 (1955), 439-454. (Chinese. English summary)

WASHINGTON UNIVERSITY,

ST. Louis, MissourI 